\title{
Effect of longitudinal magnetic field on the excess conductivity of monodomain $\mathrm{YBa}_{2} \mathrm{Cu}_{3} \mathrm{O}_{7-\delta}$ single crystals
}

\author{
K.V.Tiutierieva ${ }^{1,2}$, N.M.Zavgorodnya ${ }^{1}$, Ya.V.Dovgopolova ${ }^{1}$, \\ O.O.Chernovol-Tkachenko ${ }^{1}, R_{\text {.V.Vovk }}{ }^{1,2}$, A.Chroneos $^{3}$ \\ ${ }^{1}$ Physics Department, V.Karazin Kharkiv National University, \\ 4 Svobody Sq., 61022 Kharkiv, Ukraine \\ ${ }^{2}$ Ukrainian State University of Railway Transport, \\ 7 Feyerbaha Sq., 61050 Kharkiv, Ukraine \\ ${ }^{3}$ Faculty of Engineering and Computing, Coventry University, 3 Gulson \\ Str., Coventry CV1 2JH, United Kingdom
}

Received May 18, 2015

\begin{abstract}
In the present study influence of longitudinal magnetic field on temperature dependence of the excess conductivity in the temperature interval of transition to the superconducting state in untwinned $\mathrm{YBa}_{2} \mathrm{Cu}_{3} \mathrm{O}_{7-\delta}$ single crystals with optimal oxygen content are investigated. Causes of low-temperature "tails" (paracoherent transitions) in resistive transitions in the superconducting state are analyzed in the framework of implementation of various regimes of the phase state of vortex matter.

Keywords: excess conductivity, $\mathrm{YBa}_{2} \mathrm{Cu}_{3} \mathrm{O}_{7-\delta}$ single crystals, oxygen deficiency, pinning, 2D-3D crossover, intrinsic pinning.

Исследовано влияние продольного магнитного поля на температурные зависимости избыточной проводимости в области переходов в сверхпроводящее состояние раздвойникованных монокристаллов $\mathrm{YBa}_{2} \mathrm{Cu}_{3} \mathrm{O}_{7-\delta}$ с оптимальным содержанием кислорода. Причины появления низкотемпературных "хвостов" (паракогерентных переходов) на резистивных переходах в сверхпроводящее состояние анализируются в рамках модели реализации различных режимов фазового состояния вихревой материи.
\end{abstract}

Вплив поздовжнього магнітного поля на надлишкову провідність однодоменних монокристалів $\mathrm{YBa}_{2} \mathrm{Cu}_{3} \mathrm{O}_{7-\delta}$. Е.В.Тютерева, Н.Н.Завгороднял, Я.В.Довгополова, О.А.Чорновол-Ткаченко, Р.В.Вовк, А.Хронеос.

Досліджено вплив поздовжнього магнітного поля на температурні залежності надлишкової провідності в області переходів у надпровідний стан роздвійникованих монокристалів $\mathrm{YBa}_{2} \mathrm{Cu}_{3} \mathrm{O}_{7-\delta} 3$ оптимальним вмістом кисню. Причини появи низькотемпературних "хвостів" (паракогерентних переходів) на резистивних переходах у надпровідний стан аналізуються у рамках моделі реалізації різних режимів фазового стану вихорової матерії.

\section{Introduction}

It is well known that external magnetic field influences on the shape of temperature dependence of resistance for high-temperature cuprates in the superconducting transition [1-3]. The transition region is expanded, and the dependence of magnetoresistance $\rho(T)$ becomes nonmonotonic because of vortex dynamics $[4,5]$. The phase diagram of cuprates, which characterizes the temperature dependence of carrier concentration is parabolic dome and superconducting transition line through the pseudogap above it [6]. Between them is a region characterized by the Nernst effect, which can be interpreted as a result of the presence of vortices at temperature much higher than 
$T_{c}$. Nature of these vortices and origin of the pseudogap are still the subject of intense discussions [7-11]. At this stage in the high-temperature superconducting structures it was sufficiently well studied the behavior of the Abrikosov vortices [1]. In this case small coherence length $\xi$ and large penetration depth $\lambda$ [12] leads to the fact that pinning of these vortices in HTSC becomes effective on small-scale defects, including oxygen vacancies [4] and on the introducing impurities [13]. Twins, grain boundaries, point defects clusters strongly "fix" the vortices [14] too, which makes it difficult to study influence of oxygen vacancies on the phase state of the vortex system in high- $T_{c}$ cuprates. The layered structure of these compounds also gives rise to the so-called own pinning [14] in longitudinal magnetic fields. An important feature of high-temperature superconducting compounds $\mathrm{YBa}_{2} \mathrm{Cu}_{3} \mathrm{O}_{7-\delta}$ is that the predetermined concentration of the point defects is relatively easy to obtain by varying the oxygen content [15-17] or doping substituted elements $[18,19]$. As a result of this it can be studied the correlation between shape of the magnetoresistance curve and the concentration of the point defects.

In the present study we investigate the magnetic conductivity in untwined $\mathrm{YBa}_{2} \mathrm{Cu}_{3} \mathrm{O}_{7-\delta}$ single crystals under different values of magnetic field in $a b$-plane. Using as samples untwined single crystals we eliminate the influence of intergranural boundaries and TB allowing the selected geometry of the experiment to control the changes of contribution of the intrinsic pinning [20].

In this case, measurement of the resistivity transitions to the superconducting state, allows the investigation of the impact of the point defects to the phase state and to the vortex matter dynamics. This is achieved by analyzing the fluctuation to the conductivity that was observed in HTSC compounds at temperatures near to the critical temperature $\left(T \approx T_{c}\right)[4,13,21]$.

The aim of this work is to study the effect of a the constant magnetic field on the intrinsic pinning and the excess conductivity of single-domain single crystals of YBaCuO with optimal oxygen content.

\section{Experimental}

The $\mathrm{YBa}_{2} \mathrm{Cu}_{3} \mathrm{O}_{7-\delta}$ single crystals were grown in a gold crucible with solution-melting method, with the methodology described previously [4]. The $\mathrm{YBa}_{2} \mathrm{Cu}_{3} \mathrm{O}_{7-\delta}$ oxygen saturating regime leads to tetra-ortho structural transition that in its turn results to the crystal twinning in order to minimize its elastic energy. To obtain the untwined samples, we used a special cell at $420^{\circ} \mathrm{C}$ and pressure 30-40 $\mathrm{GPa}$, in accordance to the procedure described in [22]. To obtain homogeneous oxygen content, the crystal was annealed again in an oxygen flow at temperature of $420^{\circ} \mathrm{C}$ for seven days.

To form electric contacts the standard four-contact scheme was used. In this, silver paste was applied onto the crystal surface and the following connection of silver conductors (with diameter $0.05 \mathrm{~mm}$ ). Thereafter, they were annealed at temperature of $200^{\circ} \mathrm{C}$ in oxygen atmosphere for three hours. This methodology results in the contacts creation with resistance less than $1 \mathrm{Ohm}$ and it allows measurements with current of $10 \mathrm{~mA}$ in $a b$ plane. All the measurements were performed in temperature drift mode using the method for two opposite directions of the transport current. This effectively eliminates the parasitic signal impact. A platinum thermoresistor was used to monitor the temperature, whereas the voltage was measured across the sample and the reference resistor with B2-38 nano-voltmeters. Data from the voltmeters interface is automatically transferred to computer. The critical temperature $\left(T_{c}\right)$ was defined as the temperature corresponding to the main maximum in $d \rho_{a b}(T) / d T$ dependence in the superconductive (SC) transition.

Magnetic field at $15 \mathrm{kOe}$ was created by an electromagnet, which could vary orientation of the field relative to the crystal. Accuracy of the field orientation relative to the sample was better than $0.2^{\circ}$. The sample was mounted in the measuring cell so that the vector field $H$ was always perpendicular to the vector of the transport current $j$.

\section{Results and Discussion}

For investigations of resistive transitions in the superconducting state we used the Kouvel-Fischer method [23]. This is based on the analysis of the quantity $\chi=-d(\ln \Delta \sigma) / d T$, where $\Delta \sigma=\sigma-\sigma_{0}$ is the excess conductivity, which arises in the conducting subsystem due to fluctuation pairing of the carriers at $T>T_{c}$ [24] and determined by the phase state of vortex matter at $T<T_{c}[13$, 21]. Here $\sigma=\rho^{-1}$ is an experimentally measured value of conductivity, and $\sigma_{0}=\rho_{0}{ }^{-1}=$ $(A+B T)^{-1}$ is a term, determined by extrapolating the high-temperature linear seg- 


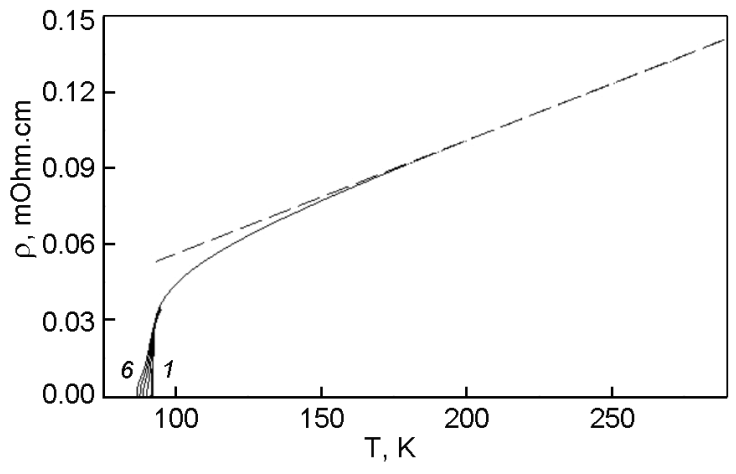

Fig. 1. Temperature dependences of resistivity $\rho_{a b}(T)$ for the $\mathrm{YBa}_{2} \mathrm{Cu}_{3} \mathrm{O}_{7-\delta}$ single crystal for $H$ $=0$ (1) and for $H=3,2 ; 6,1 ; 9,3 ; 12,4$; $15,05 \mathrm{kOe}$ (curves $2-6$, respectively). The dotted lines in the figure shows a linear extrapolation of the plots in the low temperature region.

ment up to area of the SC transition. Assuming that $\Delta \sigma$ diverges as $\Delta \sigma \sim\left(T-T_{c}\right)^{-\beta}$ at $T \approx T_{c}$, from the derivative $\chi=-$ $d\left(\ln \Delta \sigma / d T\right.$ it follows that $\chi^{-1}=\beta^{-1}\left(T-T_{c}\right)$, where $\beta$ is an indicator that depends on the dimension and the phase state of the fluctuation and vortex subsystems [13, 21, 24]. Thus, identification of the linear temperature dependence $\chi^{-1}(T)$ at the same time allows determination of important dimensional parameters and characteristic temperatures of the dynamic phase transitions in the SC carrier's subsystem.

Fig. 1 shows the temperature dependences of resistivity in the basal $a b$-plane $\rho_{a b}(T)$, measured under $H=0$ (curve 1 ) and $H=3,2 ; 6,1$; 9,$3 ; 12,4 ; 15,05$, respectively.

As can be seen from the figure, the temperature value is lowered from $300 \mathrm{~K}, \rho_{a b}(T)$ almost linearly to a certain characteristic temperature $T^{*} \approx 170 \mathrm{~K}$. Below this temperature there is systematic deviation of the experimental points down from the linear dependence, indicating that appearance of the excess conductivity $\Delta \sigma$, as discussed above. According to the modern concepts such a behavior of dependence $\rho_{a b}(T)$ at temperatures $T>T_{c}$ is conditioned by manifestation of the so-called "pseudogap anomalies" (PG), which is discussed in more detail in [25]. It should also be noted that application of magnetic field and change the magnetic field at temperatures $T>1.15 T_{c}$, within experimental error, have not effect on the behavior of the curves $\rho_{a b}(T)$, both leading to significant broadening of the superconducting transition itself, in comparison with the sharp $\left(\Delta T_{c} \approx 0.3 \mathrm{~K}\right)$ transition, observed at $H=0$. In this case, it is clear that on the tail of the superconducting transition it is observed the sharp "kink", which also appears

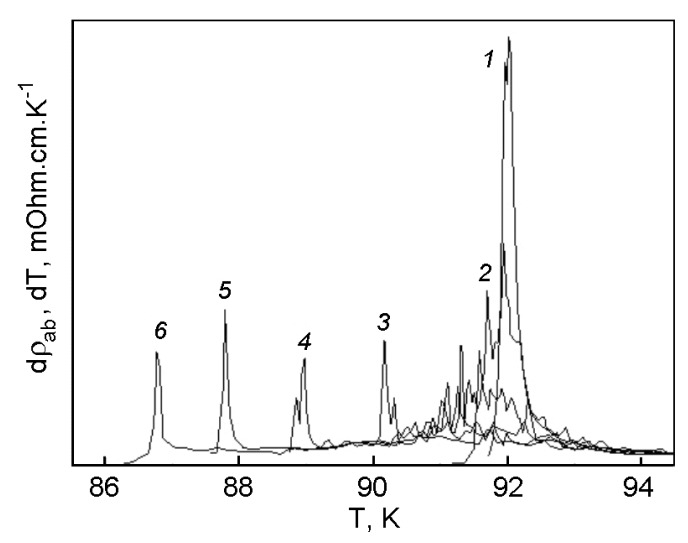

Fig. 2. Resistivity transitions to the superconducting state for the $\mathrm{YBa}_{2} \mathrm{Cu}_{3} \mathrm{O}_{7-\delta}$ single crystal in $d \rho_{a b}(T) / d T-T$ coordinates. Numbering of the curves is consistent to Fig. 1 .

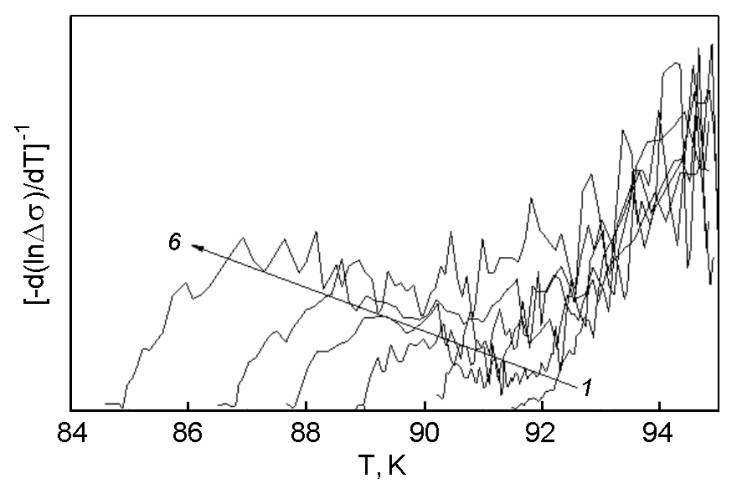

Fig. 3. Resistivity transitions to the superconducting state for $\mathrm{YBa}_{2} \mathrm{Cu}_{3} \mathrm{O}_{7-\delta}$ single crystal in $[-d(\ln \Delta \sigma) / d T]^{-1}-T$ coordinates. Numbering of the curves is consistent to Fig. 1.

in the form of the sharp low-temperature peak in the temperature dependence of the derivative $d \rho_{a b}(T) / d T$ (Fig. 2).

As can be seen from Fig. 2, the peak is present for all values of $H$, while its height increases with increasing values of $H$. According to $[4,13]$, appearance of such features in the temperature dependences $\rho_{a b}(T)$ and $d \rho_{a b}(T) / d T$ shows implementation in the first order the phase transition corresponding to melting of the vortex lattice.

Fig. 3 shows resistivity transitions to the SC state in coordinates $[-d(\ln \Delta \sigma) / d T]^{-1}-T$. In all curves in the high-temperature area of the SC transition we observe a linear area with slope $\beta \approx 0.5$, which according to [24] indicates the realization of three-dimensional (3D) regime of fluctuation carriers' existence in the system. In this, the section corresponding to the $3 \mathrm{D}$ regime is essentially unstable in magnetic field, which is consistent with the results obtained by $[4,13,21]$. When increasing the tem- 
perature from $T_{c}$ upwards, increase of the absolute value of $\beta$ occurs, this suggests the realization of 3D-2D crossover in the system [4, 13, 24]. The magnetic field application and $H$ increase lead to significant transformation of the form of the SC-transition, which is expressed in appearance of an additional lowtemperature peak, so-called "para-coherent transition". At the same time, the shift of the transition to the lower temperatures accompanies with significant increase in simultaneous amplitude and width of the peak with the $H$ increasing. Similar behavior may be due to decrease (with increase in the proportion $H$ ) the own intrinsic pinning, and thus increasing the role of the bulk pinning. This, in turn, it can contribute to transition from the ordered phase in the phase grating of the vortex so-called "vortex" or "Bragg" glass due to the accommodation of chaotic vortex pinning potential $[1,4]$. This chaotic pinning potential violates the long-range order of the vortex lattice, thereby suppressing the first-order phase transition and results to formation of glassy state of the vortices. In the resistive transitions "tails" appear, whose amplitude is less than the resistance of viscous flow $\rho_{f f}$. These are probably due to a partial pinning of the vortex liquid. In our case, oxygen vacancies can play the role of this potential [25]. In this case a the presence in the system of specific quasiparticle scattering mechanisms [26-30] and the anisotropy of a number of physical characteristics can have some influence [31].

\section{Conclusions}

In summary, the application of the constant magnetic field to $\mathrm{YBa}_{2} \mathrm{Cu}_{3} \mathrm{O}_{7-\delta}$ single crystals leads to additional para-coherent transition in the excess conductivity temperature dependences in the basic $a b$-plane in the area of resistive transition to the superconducting state. The magnetic field increase leads to simultaneous increase of amplitude and width of the peak, which corresponds to this transition and its displacement to the lower temperatures. This may be due to decrease with increasing $H$ the contribution of its own vortex pinning subsystem and the role of the bulk pinning due to presence of oxygen vacancies in the experimental sample structure. As a result, at temperatures below the critical $T<T_{c}$, the phase transition manifests the dynamic vortex liquid form - the vortex "Bragg" glass.

\section{References}

1. G.Blatter, M.V.Feigel'man, V.B.Geshkenbein et al., Rev. Mod.Phys., 66, 1125 (1994).

2. A.V.Bondarenko, V.A.Shklovskij, R.V.Vovk et al., Low Temp. Phys., 23, 962 (1997).

3. A.V.Bondarenko, A.A.Prodan, M.A.Obolenskii et al., Low Temp. Phys., 27, 339 (2001).

4. R.V.Vovk, Z.F.Nazyrov, M.A.Obolenskii et al., J. Alloys Comp., 509, 4553 (2011).

5. A.V.Bondarenko, V.A.Shklovskij, M.A.Obolenskii et al., Phys. Rev. B, 58, 2445 (1998).

6. F.Rullier-Albenque, H.Alloul, F.Balakirev, C.Proust, $E P L, 81,37008$ (2008).

7. M.V.Sadovskii, I.A.Nekrasov, E.Z.Kuchinskii et al., Phys. Rev. B, 72, 155105 (2005).

8. R.V.Vovk, N.R.Vovk, G.Ya.Khadzhai, O.V.Dobrovolskiy, Solid State Commun., 204, 64 (2015).

9. A.Solovjov, M.Tkachenko, R.Vovk, A.Chroneos, Physica C, 501, 24 (2014).

10. P.Lee, N.Nagaosa, X.Wen, Rev. Mod.Phys., 78, 17 (2006).

11. R.V.Vovk, M.A.Obolenskii, A.A.Zavgorodniy et al., J.Alloys Compd., 485, 121 (2009).

12. A.V.Bondarenko, A.A.Zavgorodniy, D.A.Lotnik et al., Fiz. Nizk. Temp., 34, 645 (2008).

13. A.A.Zavgorodniy, R.V.Vovk, M.A.Obolenskii et al., Low Temp. Phys., 36, 143 (2010).

14. R.V.Vovk, V.M.Gvozdikov, M.A.Obolenskii et al., Acta Phys. Polonica A, 121, 1191 (2012).

15. D.D.Balla, A.V.Bondarenko, R.V.Vovk et al., Low Temp. Phys., 23, 777 (1997).

16. R.V.Vovk, N.R.Vovk, O.V.Dobrovolskiy, J. Low Temp. Phys., 175, 614 (2014).

17. R.V.Vovk, N.R.Vovk, A.V.Samoilov et al., Solid State Commun., 170, 6 (2013).

18. A.Chroneos, I.L.Goulatis, R.V.Vovk, Acta Chim.Sloven., 54, 179 (2007).

19. R.V.Vovk, N.R.Vovk, O.V.Shekhovtsov et al., Supercond. Sci. Technol., 26, 085017 (2013).

20. R.V.Vovk, M.A.Obolenskii, A.A.Zavgorodniy et al., Mod. Phys. Lett. B, 25, 2131 (2011).

21. R.M.Costa, I.C.Riegel, A.R.Jurelo et al., $J$, Magn. Magn. Mater., 320, e493 (2008).

22. J.Giapintzakis, D.M.Ginzberg, P.D.Han, $J$. Low Temp.Phys, 77, 155 (1989).

23. J.S.Kouvel, M.E.Fischer, Phys.Rev., 136, A1626 (1964).

24. L.G.Aslamazov,A.L.Larkin, Fiz .Tverdogo Tela, 10, 3258 (1968)

25. R.V.Vovk, M.A.Obolenskii, A.A.Zavgorodniy et al., Low Temp. Phys., 33, 931, (2007).

26. D.H.S.Smith, R.V.Vovk, C.D.H.Williams, A.F.G.Wyatt, Phys. Rev.B, 72, 0546506 (2005).

27. R.V.Vovk, Z.F.Nazyrov, I.L.Goulatis, A.Chroneos, Physica C, 485, 89 (2013).

28. D.H.S.Smith, R.V.Vovk, C.D.H.Williams, A.F.G.Wyatt, New J. Phys., 8, 128 (2006).

29. R.V.Vovk, G.Ya.Khadzhai, O.V.Dobrovolskiy, Appl.Phys. A, 117, 997 (2014).

30. R.V.Vovk, N.R.Vovk, G.Ya.Khadzhai et al., Current Appl. Phys., 14, 1779 (2014).

31. R.Vovk, G.Khadzhai, O.Dobrovolskiy, Mod. Phys. Lett. B, 28, 1450245 (2014). 